\title{
A context-dependent representation model for explaining text repetition effects
}

\author{
GARY E. RANEY \\ University of Illinois, Chicago, Illinois
}

\begin{abstract}
The purpose of this article is to review abstract and episodic models of text repetition effects, describe the research supporting these types of models, and propose a new model called the contextdependent representation model, which can explain both abstract-like and episodic-like repetition effects. The basic assumptions of the model are that the surface form and textbase are represented in a context-independent manner, and a coherent situation model binds together the surface features and the textbase and leads to context-dependent representation. When the situation model is well developed, it limits repetition benefits to semantically or contextually similar texts. This produces contextdependent repetition effects, which are functionally similar to episodic repetition effects. When the situation model is not well developed or not task relevant, repetition benefits are not limited to contextually similar texts. This produces context-independent repetition effects, which are functionally similar to abstract repetition effects. The context-dependent representation model provides a theoretical basis for explaining past research, and it can serve as a guide for future research aimed at understanding text repetition effects as well as text comprehension and memory.
\end{abstract}

As you read this article, you will form a representation of it in memory. Suppose that you read it again. The odds are high that you will read it faster during the second reading. This is known as a text repetition effect, or rereading benefit. There is a generally accepted explanation for this repetition effect: Memory of the text formed during the first reading facilitates processing during the second reading. There is, however, intense debate regarding why and how memory facilitates rereading (e.g., Bowers, 2000; Carlson, Alejano, \& Carr, 1991; Carr, Brown, \& Charalambous, 1989; Levy \& Burns, 1990; Levy et al., 1995; Masson, 1995; Tenpenny, 1995). The debate has been centered on whether text repetition effects are based on abstract or episodic text representations and how the reader may use such representations. Resolving this debate is important because it will enhance our understanding of how texts are represented in memory.

To explain text repetition effects, I propose a model that integrates the notion of context-independent and contextdependent representations with components of current text comprehension models. I will show how contextindependent representations can be used to explain what has classically been defined as abstract repetition effects, and how context-dependent representations can be used to explain what has been defined as episodic repetition

Portions of the research reported here were supported by grants from the National Science Foundation (SBR-972448) and from the Campus Research Board at the University of Illinois at Chicago. I am grateful to Betty Ann Levy, Michael Masson, Bette Bottoms, David Therriault, Scott Minkoff, Jeanette Altarriba, Gabriel A. Radvansky, and Keith Rayner for comments on this paper. Correspondence should be addressed to G. E. Raney, Department of Psychology (M/C 285), University of Illinois at Chicago, Chicago, IL 60607 (e-mail: geraney@uic.edu). effects. In simple terms, the questions addressed here are as follows: What is it about memory that makes a text easier to read a second time? And, how can memory for one text make a second text easier to read? I begin by describing past research. Following this I describe the proposed model, specify its predictions, illustrate the model's utility in resolving discrepancies in past research, and describe future research needed to evaluate the model.

Most of the research described here is based on a text repetition procedure: Individuals read a text and then read a version of the text again. To examine how perceptual, lexical, and conceptual features of a text influence memory, features of the text are systematically manipulated between the first and second readings so that the text is read in the same or different form and in the same or different context. The size of the repetition effect is then used as an index of how much the first encounter facilitates processing during the second encounter. Repetition effects thus serve as implicit measures of memory (Carr et al., 1994; Levy, 1993; Raney \& Rayner, 1993, 1995; Rayner, Raney, \& Pollatsek, 1995).

Repetition effects are sometimes described as transfer benefits if the difference between the first text and the second text is substantial. For example, assume a reader reads a text about plants and then reads a text about farming. If reading about plants helps the reader process the text about farming, then information in memory about the plants passage is described as transferring to the farming passage. Transfer benefits might occur if the two passages share words or ideas; therefore, transfer benefits can be viewed as repetition effects based on words or concepts.

Repetition facilitates word processing in tasks other than reading extended text, including word naming 
(Masson \& Freedman, 1990; Scarborough, Cortese, \& Scarborough, 1977), word similarity judgments (Woltz, 1990), lexical decision (Masson \& Freedman, 1990; Rugg, 1990), semantic classification (Monsell, 1985, 1987), and perceptual identification tasks (Humphreys, Besner, \& Quinlan, 1988; Jacoby, 1983; Rueckl, 1990). But there is more to text repetition effects than simply faster processing of individual words. Rereading can also lead to increased comprehension and enhanced memory for meaning (Goldman \& Saul, 1990; Levy, 1983; Levy \& Begin, 1984; Levy, Di Persio, \& Hollingshead, 1992; Levy \& Kirsner, 1989; Millis \& Simon, 1994; Raney, 1993), but note that faster word processing alone can be dissociated from enhanced comprehension (Bourassa, Levy, Dowin, \& Casey, 1998; Levy, Abello, \& Lysynchuk, 1997). In this article, I focus on repetition effects for texts as opposed to isolated words. Tenpenny (1995) and Bowers (2000) have provided excellent reviews of word repetition effects.

\section{ABSTRACT VERSUS EPISODIC MODELS OF TEXT REPETITION EFFECTS}

Although text repetition effects are common, the source of the facilitation remains unclear. The two dominant explanations have been labeled abstract and episodic accounts (Tenpenny, 1995). Supporters of the abstract view argue that repetition effects result from primed, abstract word representations, such as the "logogens" proposed by Morton (1969). Reading a word once activates its logogen and this makes the word easier to activate a second time (over limited durations). Because the logogen is an abstract, lexical-level representation, it does not matter exactly how the logogen is activated. In an extreme form, the abstractionist view predicts that repetition effects should occur independent of linguistic context or modality (Carr et al., 1994; Tenpenny, 1995). Simply put, reading a word once should cause the word to be read faster during a second encounter even if the word appears in a new linguistic context. For example, if a passage refers to a black cow at one point and then later refers to a brown cow, the word cow should be easier to process the second time even though the word cow refers to a different cow and is presented in a new context. This type of repetition benefit is described as being mediated at the word level to indicate that the representation of the word itself controls text repetition effects, not the relationship between a word and its context.

Two aspects of the abstract view need emphasis. First, according to the prior definition, abstract repetition benefits accrue from facilitated processing within the lexicon. This can be due to heightened activation of a lexical entry or to changes in structure that make activation of a lexical entry easier on subsequent encounters (e.g., lowering the activation threshold of a word; Morton, 1979). Consequently, abstract repetition effects are not dependent on what a reader remembers about a text or how the reader represents the text. Postulating changes in the structure of the lexicon also provides a mechanism by which abstract representations could produce longterm repetition effects (Bowers, 2000). Second, facilitated (faster) lexical processing can lead to repetition benefits that are not lexical in nature. As an example, Levy et al. (1997) and Bourassa et al. (1998) demonstrated that repeated exposure to individual words eased processing demands associated with those words when they were part of a subsequently read text, thereby supporting enhanced comprehension of the text by freeing up resources normally devoted to lexical processing. The important point is that even according to purely lexical models, repetition can facilitate nonlexical processes.

Advocates of the episodic view argue that repetition effects are due to memory for specific events or information within a text (i.e., episodes). Reactivating the initial episode during a repeated reading facilitates reprocessing of the text. Defining the exact nature of an episode is difficult because anything that occurred in the text can be part of the episode. Episodes can incorporate the meaning of a passage, details about the words used in the passage, the precise linguistic context, the processes used to comprehend the passage, and even physical parameters such as font. Given that most anything can be a part of an episode, content alone cannot be used to define an episode. In text repetition studies, the key feature of an episode is that it is a single unit that tightly links together the contents (Levy \& Burns, 1990; Levy et al., 1995; Tenpenny, 1995). This is referred to as contextbound memory. Because context is so critical, the more similar two texts are on each occurrence, the more transfer there will be from one text to the next. This occurs because similar texts will recruit elements of the same episode. Reading two dissimilar texts should not lead to repetition effects because the second text cannot reinstate the episodic representation of the first text. Unlike abstract repetition effects, which are not based on memory for the text per se, episodic repetition effects are dependent on memory for the information in a text or the processes used to comprehend the text (Kolers, 1975, 1976; Levy et al., 1995; Masson, 1995).

In terms of the black cow example mentioned above, the episodic memory for the first occurrence of the word cow might include black as part of the local context. Thus, reading about the black cow would not facilitate processing of the word cow in the phrase brown cow because cow appears in a different context and has a different referent. The elements of the episode referring to the cow do not match, so there should be no repetition effect. If black cow was read twice, the second occurrence would be read faster because it matches the first instance. O'Brien, Raney, Albrecht, and Rayner (1997) found this pattern of results.

From an experimental perspective, the most important outcome for defining whether repetition effects are abstract or episodic has been whether rereading benefits transfer across linguistic contexts. This is an effect-driven definition. Repetition benefits that transfer across linguistic contexts are generally labeled abstract even if the source of the effect is not known to be lexical or word- 
level facilitation. Experiments that produce no transfer benefits or produce reduced transfer benefits across changed contexts relative to matched contexts are generally taken as evidence for the episodic view. This reflects the theoretical dichotomy of past research: Repetition effects are described as abstract or episodic, but not both. One reason for using an effect-driven definition is that a single experimental procedure can produce diametrically opposed results as a function of task demands, such as instructions (Carlson et al., 1991), and subject variables, such as reading ability (Faulkner \& Levy, 1994, 1999). A problem with this effect-driven classification system is that evidence for episodic transfer is sometimes based on the lack of evidence for abstract transfer (e.g., no transfer for mismatched contexts) or on the existence of larger repetition effects for matched contexts than for mismatched contexts. Neither of these conditions eliminates the possibility that abstract transfer benefits exist. The following review illustrates these points.

\section{TEXT REPETITION EFFECTS}

Kolers's classic studies involving reading transformed (e.g., inverted) typescript jump-started research on this topic. He demonstrated repetition sensitivity to perceptual factors in several experiments (Kolers, 1975, 1976; Kolers \& Ostry, 1974). When sentences were initially read in transformed typescript, repetition effects were largest when the rereading was in the same transformed typescript (matched) as opposed to a different typescript (mismatched). Kolers interpreted these results in terms of enhanced perceptual fluency and recall of the processes used to analyze the transformed typescript. He concluded that these results supported episodic representation because repetition effects were reduced when the typescript (perceptual context) did not match on each encounter.

Kolers's work is regularly cited as strong evidence for the episodic view, but several researchers have failed to fully replicate his findings. For example, Jacoby, Levy, and Steinbach (1992) did not find sensitivity to perceptual changes across readings when subjects read texts out loud, but did when comprehension was the primary task. Jacoby et al. suggested that the initial processing episode was not fully reinstated when the task emphasized perceptual processes (as in reading aloud) and that this limited sensitivity to changes in perceptual features. Horton (1985) and Tardif and Craik (1989) found similar magnitude repetition effects for texts that were presented in matched or mismatched typescripts. Each of these authors concluded that comprehension processes drive repetition effects, not enhanced perceptual fluency. Recent work by Masson (1995) supports this explanation. Although the results and conclusions of these studies differ from those of Kolers, they converge on an episodic view of text repetition effects. But also note that repetition effects were maintained across changed perceptual contexts under certain conditions. If a portion of these perceptually context-independent repetition effects resulted from enhanced lexical processing, such as eased reading of transformed typefaces, or by-products of enhanced lexical processing, such as freeing up resources for comprehension processes, then a portion of these repetition effects might be consistent with the abstract view.

Carr et al. (1989) tested the abstract and episodic theories by manipulating linguistic context between readings. They had subjects read a text in normal form or with word order scrambled, then reread the same text (matched) or the opposite version (mismatched). Repetition effects occurred in each condition and were independent of the type of text read during the first reading. Carr et al. (1989) concluded that context was relatively unimportant. Simply activating words during the first reading facilitated reprocessing of the words as part of a text. This outcome provides evidence for lexically based, abstract transfer benefits that are not dependent on precise repetition of the original context.

Other studies initially cast doubt on Carr et al.'s (1989) findings. For example, Levy and Burns (1990, Experiment 3) repeated Carr et al.'s (1989) experiment, but found little or no repetition effects. However, there were important procedural differences between the studies. Levy and Burns instructed readers to read silently and focus on the meaning (even for scrambled texts), whereas Carr et al. (1989) instructed readers to read aloud as accurately as possible (placing emphasis on individual words) and ignore meaning. To examine task differences, Carlson et al. (1991) used the same paradigm and instructed subjects either to ignore the meaning of the texts or to focus on the meaning of the texts. When subjects were instructed to ignore meaning, Carr et al.'s (1989) results were replicated. When subjects were instructed to read for meaning, Levy and Burns's results were obtained. This demonstrates that the apparent source of repetition effects may be substantially altered by task demands and attentional focus (see also Carr \& Brown, 1990; Jacoby et al., 1992; Salasoo, 1986). As a result, one must be cautious when interpreting studies that use different methodologies and instructions, or that compare a combination of text types, such as normal versus scrambled texts or inverted versus normal texts, because the processing requirements might differ for each text (Carr \& Brown, 1990; Whittlesea, 1990). Overall, the studies by Carr et al. (1989), Carlson et al., and Levy and Burns support the conclusion that repetition effects can be either abstract or episodic in nature.

Levy and colleagues have conducted several rereading studies using nonscrambled texts. In Levy et al. (1992, Experiment 3), subjects read normal texts five times in succession while proofreading for spelling errors. During the fifth reading, a few words in half of the passages were replaced by synonyms. Reading times for sentences containing synonyms were slower than reading times for the sentences without synonyms, but both conditions showed reliable repetition effects. That is, paraphrases produced partial transfer benefits. Levy, Barnes, and Martin (1993) used the paraphrase method without the proofreading task. They examined rereading benefits for paraphrases in which syntactic structure was 
altered but wording was preserved, or wording was altered using synonyms but syntactic structure was preserved. They found significant repetition effects in both conditions, but slightly larger benefits for syntactic paraphrases. Levy et al. (1995, Experiments 4 and 7) extended the paraphrasing method by examining transfer across texts (i.e., how reading one text influences reading a second text). Second readings were either related texts (successive sections of a novel with many overlapping words), paraphrases of the related texts (words from the related texts were replaced by synonyms), different texts (new stories with many overlapping words from the first story), or unrelated stories (new stories with few overlapping words from the first story). Only related and paraphrased texts produced reliable repetition effects. ${ }^{1}$

In all three of the studies just mentioned, Levy and colleagues concluded that their results support the episodic view because changing the local context reduced the repetition effect. They also concluded that meaning overlap, not word overlap, produced the repetition effects and that meaning overlap led to a reinstatement of the episode created during the first reading. These results are not, however, entirely inconsistent with the abstract view because paraphrases formed from synonyms produced significant repetition effects. Thus, partial repetition benefits were found even though the linguistic context was changed. This leaves open the possibility that that some type of abstract representation contributes to the repetition effect for paraphrased texts.

In a similar experiment by Raney and Rayner (1995), subjects read texts twice in succession while their eye movements were monitored. Recording eye movements allows examination of overall reading times as well as individual word reading times. The second readings were either identical or had one word changed to a synonym. Both repeated words and synonyms were read faster during the second reading. Importantly, reading times for synonyms and repeated words were similar. Raney and Rayner concluded that their results support the abstract view. They suggested that the activation of the meaning referenced by a word, not just the word itself, was enhanced. Because a target word and its synonym accessed common concepts, reading times for synonyms were facilitated. This is a slightly different interpretation of the abstract view. According to the classic definition of the abstract view, facilitation results from heightened activation of a particular word, which makes reactivation easier (Carr et al., 1989; Tenpenny, 1995). In this sense, words are strongly linked to their meanings, which is one reason why this is referred to as word-level transfer. Raney and Rayner (1995) called into question whether abstract repetition effects should be defined as facilitated processing of a specific word (i.e., the lexical entry itself), the underlying concept referred to by a word, or both the word and its concept. A recent study by Raney, Therriault, and Minkoff (2000) provides a starting point to address this issue.

Raney et al. (2000) had subjects read a set of short texts twice in succession while monitoring their eye movements. The passages were either identical during the second readings or paraphrases (formed using synonyms). For overall reading time, repetition effects were found for identically repeated texts and for paraphrases, but the effect was slightly larger for repeated texts. Recall that Levy and colleagues (Levy et al. 1993; Levy et al., 1995; Levy et al., 1992) found the same pattern of results. Raney et al.'s (2000) analyses of fixation times on individual words revealed an interesting difference between repeated words and synonyms. Repetition effects were found for repeated words and synonyms, but reduced fixation durations on synonyms were largely confined to words that were fixated more than once. Repeated words were read faster regardless of whether they were fixated once or twice. Multiple fixations are often made when a word is difficult to integrate into its context (Rayner \& Pollatsek, 1989), so this finding indicates that reduced fixation duration for synonyms was probably a function of reduced integration difficulty. The eye movement data support the conclusion that early stages of word processing, such as lexical access, and later stages of processing, such as integration, are both facilitated for repeated words, but only later stages of processing are facilitated for synonyms.

Raney et al.'s (2000) experiment shows that repeating the orthographic features of a word provides a benefit that is separable from repeating the meaning of the word, and that the source of repetition benefits may be the lexical entry itself of the meaning referred to by a word. Once again, the data may be interpreted using an abstract model or an episodic model. The existence of significant rereading benefits for paraphrased passages supports the abstract view because facilitation occurs across changed linguistic contexts (i.e., when words are changed to synonyms). Differences in fixation times for identical words and synonyms support the episodic view because precisely repeating the linguistic context (i.e., using identical words) produced greater benefit than that obtained when the linguistic context was altered (i.e., using synonyms).

A potential limitation of the paraphrase method is that readers sometimes report noticing differences between texts, which might focus attention on wording, reduce rereading benefits for synonyms, and lead to slower reading for paraphrases. Raney, Atilano, and Gomez (1996) avoided this problem by presenting bilingual readers with translations during the second reading. Translations are useful because they are naturally expected to contain different words even though meaning is preserved. Because fluent bilinguals share conceptual representations across languages but maintain distinct lexical representations (Altarriba, 1992; Kroll \& de Groot, 1997), presenting bilinguals with different language texts during a first and second reading provides a natural way to manipulate lexical features while repeating conceptual features (Altarriba, Kroll, Sholl, \& Rayner, 1996).

In Raney et al. (1996), Spanish/English bilinguals read texts twice in succession while their eye movements were recorded. The second reading was either the same passage in the same language (identical) or the translation. 
Embedded in each passage were sets of cognates and noncognates. Cognates share perceptual and conceptual representations across languages (e.g., the English word problem and the Spanish word problema), whereas noncognates share only conceptual features (e.g., issues, asúntos) (de Groot \& Nas, 1991). If repetition benefits result purely from conceptually based facilitation, then rereading benefits should be similar for cognates and noncognates. If a portion of across-language repetition effects results from the reinstatement of lexical items via the repetition of perceptual features, then rereading benefits should be larger for cognates than for noncognates. Raney et al. (1996) found that overall reading times and fixation durations (gaze durations) decreased during the second readings for same-language passages and for translations, although fluency level moderated effect sizes. For fluent bilinguals, fixation times on identical words (same language), cognates, and noncognates were reduced by similar amounts. For nonfluent bilinguals (individuals with 2 or 3 years of Spanish training), fixation times on identical words, cognates, and noncognates were similar when the second reading was in English (their dominant language). When the second reading was in Spanish, nonfluent bilinguals read identical words and cognates faster than noncognates.

Raney et al.'s (1996) bilingual study supports several conclusions. Most generally, it illustrates that word meanings are not necessarily tied to individual words, the precise local context, or the language used in a text (see also Raney, Obeidallah, \& Miura, 2002). This supports the separation of concept-based and word-based (i.e., repeating the exact word) sources of abstract transfer benefits described by Raney and colleagues (Raney et al., 2000; Raney \& Rayner, 1995). ${ }^{2}$ Even so, this study can be interpreted as supporting the episodic position. Because translations refer to the same events and situations as the original texts, repetition effects could be based on reinstatement of the original episodes without reference to specific words (i.e., higher level semantic processes were facilitated, but lexical processing was not facilitated). It appears that neither abstract nor episodic models provide a complete explanation of the data.

\section{A CONTEXT-DEPENDENT REPRESENTATION MODEL OF TEXT REPETITION EFFECTS}

Given the ample evidence supporting the existence of both abstract and episodic repetition effects, the most appropriate conclusion may be that both abstract-like and episodic-like repetition benefits exist. Despite the wealth of evidence, past research generally treats abstract and episodic repetition effects as mutually exclusive. As an alternative to the abstract-episodic dichotomy, I propose a context-dependent representation model in which memories vary in their degree of context dependence. ${ }^{3}$ According to the model, context-independent representations produce abstract-like repetition effects and contextdependent representations produce episodic-like repeti- tion effects. The concept of context-independent and context-dependent memory is applied directly to text representation by postulating different degrees of context dependence in memory for the surface form, the textbase, and the situation model (van Dijk \& Kintsch, 1983).

Because the context-dependent representation model builds on van Dijk and Kintsch's three levels of text memory, a brief summary of their model is warranted. Van Dijk and Kintsch (1983) suggested that texts are represented at three distinct levels: the surface form, the textbase, and the situation model. The surface form contains the wording used in the text. Surface-level representations result from lexical and syntactic analysis of the text (Fletcher \& Chrysler, 1990; van Dijk \& Kintsch, 1983). The textbase represents the text's meaning as a network of propositions (Kintsch, 1974; Kintsch \& van Dijk, 1978). The textbase is the result of processes used to create the semantic content of the text, but it is not dependent on the background knowledge used to create the semantic representation. According to Fletcher and Chrysler (1990), the textbase "corresponds to the equivalence class of all content- and structure-preserving paraphrases of a text" (p. 175). This is important for interpreting results from text repetition studies that modify a text's wording or syntactic structure between readings. The situation model is an integration of the textbase and prior knowledge, and can be thought of as a description of the events and actions (episodes) that are described by the text. The situation model goes beyond the contents of the text itself and includes general knowledge, inferences, and other products of text comprehension (Fletcher, 1994; Kintsch, 1988; Kintsch, Welsch, Schmalhofer, \& Zimny, 1990). Situation models are often described as similar to Johnson-Laird's (1983) concept of mental models, although Johnson-Laird did not originally apply mental models to text comprehension. Current theories of text comprehension describe the construction of a situation model as one of the reader's primary goals (Gernsbacher, 1990; Graesser, Millis, \& Zwaan, 1997; Johnson-Laird, 1983; Kintsch, 1998; van Dijk \& Kintsch, 1983; see Zwaan \& Radvansky, 1998, for a review of situation models in language).

The context-dependent representation model extends van Dijk and Kintsch's (1983) theory by specifying that the three levels of representation reflect different degrees of context dependence. The basic assumptions of the model are that the surface form and textbase are represented in a context-independent manner and that a welldeveloped situation model binds together the surface form and textbase, thereby creating context-dependent representation. Because the situation model acts to bind together elements of the text representation, task demands (e.g., instructions), processing strategies (e.g., read for meaning), characteristics of the texts (e.g., text coherence), and reader characteristics (e.g., reading ability) that influence situation model development will also influence the degree of context dependence and, consequently, the magnitude of text repetition effects and transfer benefits. As defined below, context-independent 
representations support repetition effects that transfer across linguistic contexts. Context-dependent representations are, as the name implies, bound to their original context; therefore, repetition effects are restricted to similar linguistic contexts.

How might this be? In essence, the situation model binds the surface features and the textbase to the text representation, thereby creating a tightly integrated memory structure. The stronger the integration of the memory structure, the greater the degree of context dependence because the representation becomes more unitized. Others have described the situation model as the glue that holds together the contents of memory (Graesser et al., 1997; Zwaan \& Radvansky, 1998). This description is appropriate here too. The more complete the situation model, the more tightly glued the surface features and textbase become to the text representation. Context-dependent representation may also be thought of as similar to the concept of state-dependent learning. In state-dependent learning, information acquired in one state or context is difficult to process or retrieve within a new context. Establishing a well-developed situation model leads to context-dependent representations, which, in turn, lead to context-dependentrepetition effects. Context-dependent repetition effects are analogous to episodic repetition effects in that repetition benefits are restricted to semantically similar contexts.

If there is no situation model or a poorly developed situation model, then the surface features and the textbase are not tightly bound or glued to the text representation. This creates context-independent representations. Contextindependent representation may be thought of as similar to state-independentlearning. In state-independentlearning, information acquired in one state or context can be easily processed or retrieved within a new context. Because context-independent representations are not bound to their original context, repetition benefits can occur across varied linguistic contexts. This is analogous to abstract repetition effects.

An important point is that processes that lead to contextindependent and context-dependent representations are not turned on or off. An attempt is always made to develop some type of situation model, and in the absence of a situation model, the surface features and textbase are represented in a context-independentmanner. What varies is the degree of context dependence. Task demands, processing strategies, text characteristics, and reader characteristics influence the development and application of situation models, and this determines the degree to which a text representation will be context independent or context dependent. I will return to this issue when describing the predictions of the context-dependentrepresentation model.

Note that the context-dependent representation model is not equivalent to proposing that abstract and episodic representations simply coexist in memory. The contextdependent representation model differs qualitatively from the abstract and episodic models. Several important differences are worth highlighting. First, the source of repetition benefits is substantially different. Prior models generally describe the source of abstract repetition effects as enhanced lexical activation of individual words or structural changes in the lexicon and the source of episodic repetition effects as the reactivation of a specific memory (e.g., Carr et al, 1989; Levy et al., 1993). In the context-dependent representation model, abstractlike and episodic-like repetition effects result from differences in the degree of context dependence of information in memory, and this determines whether information from one text can influence processing of a second text. Neither the activation level nor the retrievability of concepts determines the level of context dependency. Second, the model merges context-independent and contextdependent information into a single memory, and both are involved in producing text repetition benefits. Thus, context-independent and context-dependent memories are explicitly defined as compatible, whereas repetition effects based on abstract and episodic representations historically have been defined as incompatible. Third, context dependence is a continuum, whereas abstract versus episodic representation is a dichotomy. Defining the source of repetition effects as a continuum allows seemingly distinct patterns of repetition effects to be explained using a single mechanism, which creates a more parsimonious model than the dichotomous approach present in abstract and episodic explanations. Although the context-dependent representation model differs from abstract and episodic models, the notion of contextindependent and context-dependent repetition effects is consistent with the effect-driven descriptions of results found in prior text repetition studies. This allows the model to be easily tied to the outcomes of past research.

There is evidence for the model's primary assumptions. Several studies support the existence of contextindependent representation of the surface form and textbase. In the study by Levy et al. (1993) described earlier, subjects read a text and then either read a paraphrased version of the text that changed the syntactic structure but used the same words, or a paraphrase that changed the wording but maintained the syntactic structure. Repetition effects were found for both types of paraphrases. Raney et al. (2000) found the same pattern of results using wording paraphrases. Also recall that Raney et al. (1996) found large repetition benefits when fluent bilinguals read translations during the second reading. Of relevance here is that paraphrases and translations substantially alter the surface form and textbase, but the situation model is basically unchanged. If repetition benefits reflect only the reinstatement of an existing episode or situation model, then repetition benefits for identical texts, paraphrased texts, and translations should be similar because the same situation is being reinstated. Changing the surface form and textbase reduces the magnitude of repetition benefits, but does not eliminate repetition benefits. This implies that repetition benefits due to repetition of the surface form, textbase, and situation model are separable. Furthermore, these studies demonstrate that the surface form and textbase do not need to be repeated identically to produce repetition effects. That is, 
when the situation model is well formed and repeated, the surface form and textbase can be altered without eliminating repetition effects. This indicates context-independent representation of the surface form and textbase.

There is also support for the assumption that the situation model binds together the contents of text memory. Raney, Minkoff, and Therriault (1997) conducted a study to determine whether words (surface form) from one passage will facilitate processing of the same words in a second passage when the situation models do or do not overlap. The readers' eye movements were monitored while they read pairs of interleaved text passages (see Glanzer \& Nolan, 1986). ${ }^{4}$ In interleaved reading, a reader simultaneously reads two text passages (e.g., Passage A and Passage B). The reader reads Sentence 1 of Passage A, then Sentence 1 of Passage B, then Sentence 2 of Passage A, then Sentence 2 of Passage B, and so on until the end of each passage. Two conditions are relevant. In the semanticoverlap condition, subjects read passages about two different topics (e.g., evolution and hybrid plants) that shared a few themes (e.g., the creation of new species). Sharing themes provides situation model overlap. In the nosemantic-overlap condition, subjects read passages about two unrelated topics (e.g., The Muppet Show and hybrid plants) that did not share themes. A set of target words was used once in each passage (i.e., a target was used in Passage A and then in Passage B). According to the contextdependent representation model, target words in Passage A will facilitate processing (i.e., reduce fixation durations) of the same words in Passage B if the situation models overlap (the semantic-overlap condition) but not if the situation models do not overlap (the no-semantic-overlap condition). Precisely this pattern of results was found. This supports the assumption that the situation model binds information to the original context, which, consequently, restricts repetition benefits to conceptually similar texts.

More recently, Levy (2001) directly examined how situation models influence repetition benefits. In a clever series of experiments, Levy (2001) had subjects read texts that were very difficult to understand without a title, but easy to understand when a title was provided (modeled after Bransford, 1979). In essence, individual words and phrases (i.e., the surface form and textbase) were easy to comprehend without a title, but developing a coherent situation model was very difficult. The subjects read identical passages twice, either with or without a title on each reading. Four conditions were included. In the title/title condition, subjects read the passages with the title presented before each reading. In the notitle/no-title condition, subjects read the passages twice without the title. In the no-title/title condition, subjects were not given the title before the first reading but were given the title before the second reading. In the title/notitle condition, subjects were given the title before the first reading but not before the second reading.

Several findings from Levy's (2001) study are of particular interest. First, rereading benefits were larger for the title/title condition than for the no-title/no-title condition or the no-title/title condition. This demonstrates that rep- etition benefits are larger when the surface form, textbase, and situation model match across readings (title/ title) than when only the surface form and textbase match across readings (no-title/title, no-title/no-title). That is, creating a situation model enhances repetition benefits. Second, when no title was presented before the second reading (title/no-title, no-title/no-title), reading time per sentence during the second reading was similar across the first 10 sentences. This suggests that the situation model formed during the first reading was not initially recruited in either condition. For Sentences 15-25, reading time for the title/no-title condition was faster than for the no-title/ no-title condition. This suggests that the situation model formed during the first reading in the title/no-title condition was eventually recruited, which, in turn, facilitated processing during later portions of the texts. Thus, initially the title/no-title and no-title/no-title conditions showed similar rereading benefits. This is expected if reprocessing benefits were initially mediated by surface-level and textbase-level similarity. As reading continued, the situation model from the first reading of the title/no-title condition became available and facilitated rereading. Levy's (2001) study is consistent with the conclusion that the situation model binds together the contents of text memory.

There is also evidence that task differences that influence the format of the text representation will alter the degree of context dependence. For example, tasks that emphasize wording, such as reading aloud and trying to correctly pronounce the words, enhance memory for surface form and support context-independent repetition benefits (Carlson et al., 1991; Carr et al., 1989; Carr et al., 1994; Jacoby et al., 1992). Tasks that emphasize meaning, such as reading for comprehension, enhance memory for the situation model and more readily support contextdependent repetition benefits (Carlson et al., 1991; Levy \& Burns, 1990; Jacoby et al., 1992).

Differences in reading ability also influence how a text is represented. For example, good readers develop more complete situation models than do poor readers or beginning readers; therefore, good readers will form more context-dependentrepresentations. One reason this occurs is that poor and/or beginning readers have difficulty processing surface- and textbase-level information, which limits the amount of resources available for processing higher level information (Faulkner \& Levy, 1994; Long, Oppy, \& Seely, 1997; Oakhill, 1994; Rashotte \& Torgesen, 1985; Samuels, 1979). Faulkner and Levy (1994) found that second-grade children showed transfer benefits for pairs of texts that shared wording (surface form) but not semantic content. In contrast, sixth-grade children who read the relatively easy second-grade texts showed transfer benefits only for text pairs that shared semantic content. Thus, transfer benefits were context independent for the second-grade children but were context dependent for the sixth-grade children. Good readers also form more globally coherent (integrated) representations than do poor readers (Long \& Chong, 2001; Oakhill, 1994), which would also lead to a higher degree of context dependence for good readers. 
As noted above, the context-dependent representation model extends current models of text representation and text repetition effects to create a new model. What is new is the explicit use of context dependency as a mechanism for explaining abstract-like (context-independent) and episodic-like (context-dependent) repetition effects; the assumption that the surface form, textbase, and situation model vary in their degree of context dependence; and the assumption that the situation model binds together surface features and the textbase to create contextdependent memories. Combining these properties creates a powerful model that can explain many of the seemingly inconsistent results produced by past research. This is a parsimonious model because context-independent and context-dependent repetition effects can be produced by a single mechanism. This is also a flexible model in that tasks or processes that alter the degree of context dependence can alter the magnitude of both context-independent and context-dependent repetition effects. Most importantly, the model provides a theoretical basis for predicting when the surface form, the textbase, and the situation model will support repetition effects and transfer benefits.

\section{PREDICTIONS OF THE CONTEXT- DEPENDENT REPRESENTATION MODEL}

The structure of the context-dependent representation model leads to two general predictions about when and how text repetition effects will occur: (1) When a coherent situation model is available in memory and task relevant, it will serve as a retrieval guide, and processing of situation-consistent information will be facilitated (i.e., repetition effects will appear to be based on contextspecific episodes); (2) when a coherent situation model is not available in memory or not task relevant, retrieval will be guided by the surface form and textbase, and elements of the surface form and textbase that are repeated will be facilitated (i.e., repetition effects will appear to be based on abstract, context-independent information).

The basic predictions of the context-dependent representation model are illustrated in Figure 1. The first thing to notice is that repetition effects are not predicted to be all or none, but graded. This reflects the fact that texts vary in their degree of overlap of surface forms, textbases, and situation models. In particular, graded repetition effects reflect several qualities of situation models, including the following: (1) Situation models are not formed in an all-or-none manner (Zwaan, Langston, \& Graesser, 1995; Zwaan \& Radvansky, 1998), (2) elements of the situation model possess varied accessibility levels (Glenberg, Meyer, \& Lindem, 1987; Gernsbacher, 1990; O'Brien \& Albrecht, 1992; Singer \& Halldorson, 1996; Zwaan, 1994; Zwaan et al., 1995), (3) the reader's goals (via instructions) and background knowledge influence situation model development (Singer \& Halldorson, 1996; Zwaan, 1994), and (4) situation models may not be needed (are not relevant) in all tasks (Singer \& Halldorson, 1996; Zwaan \& Radvansky, 1998). The implication is that repetition effects will vary on the basis of the situation model's contents and completeness, and on task demands. A well-developed situation model should create a stronger conceptual bond with the surface features and the textbase than would a poorly developed situation model. The second thing to notice about Figure 1 is that intermediatesized repetition effects are predicted when the situation model is not task relevant or cannot be well formed (bars C and D). Under these conditions, repetition effects must be based primarily on overlap of surface features and the textbase. Repetition effects will again vary in size as a function of task demands.

According to the model, the greater the similarity between the situation models of two texts, the larger the expected repetition effect. This is a representation-based approach. That is, if the representations are similar, then repetition effects should occur. Because task demands influence how a text is processed and represented, processing strategies also influence repetition effects. Specifically, if the processes used to create a situation model for one text overlap with the processes used to create a situation model for a second text, then the situation models are likely to be more similar than if the processes do not match. Processes that enhance the degree of situation model overlap will, consequently, enhance repetition benefits. Similarity may be defined at a conceptual level (i.e., processing similar concepts) or a task level (i.e., reading two texts in the same manner). The same reasoning may be applied to the surface form and textbase: Processes that enhance the similarity of the representations of the surface form and textbase will also enhance repetition benefits. In this manner, the contextdependent representation model is compatible with the basic assumption of transfer-appropriate processing models when applied to text repetition (e.g., Levy et al., 1992; Masson, 1995; Roediger, 1990).

Bars A and B of Figure 1 represent conditions in which situation models are well formed and task relevant. Here the prediction is straightforward: Repetition effects will occur if the texts' situation models overlap. This is essentially equivalent to the conclusion that repetition effects result from meaning overlap (Levy et al., 1993; Levy et al., 1995; Levy, Masson, \& Zoubek, 1991; Masson, 1995). The error bars indicate that repetition effects will vary in size as a function of the completeness of the situation models and the amount of overlap between texts. The most variability should occur when there is situation model overlap (bar A) because the degree of overlap can vary at each level of representation (Zwaan et al., 1995). Repetition effects should increase as the overlap between situation models, surface forms, and textbases increases (Levy, 2001; Raney et al., 2000). The least variability should occur when situation models are well developed but there is no semantic overlap between them (bar B). In this case, little or no repetition benefit is expected. When situation models do not overlap, overlapping surface forms and textbases will not produce repetition effects (Levy et al., 1995; Raney et al., 1996). This does not mean that context-independent information is not contained in memory, only that it is 


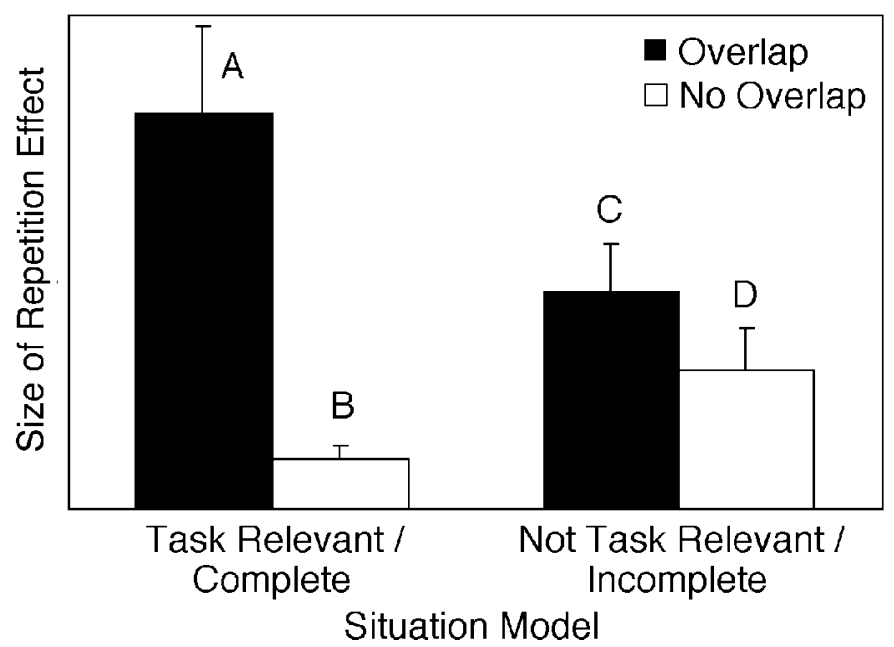

Figure 1.Predicted repetition effects based on the context-dependent representation model. The figure is intended to indicate the pattern of repetition effects, not the absolute magnitude of repetition effects. Tasks are classified according to whether situation models are task relevant and complete, or not task relevant and incomplete. Overlap and no overlap indicate whether texts share elements of their situation models. Error bars indicate expected variation in repetition effects as a function of the degree of overlap of surface forms, textbases, and situation models between texts. Bars are labeled $A, B, C$, and $D$ for reference within the text.

constrained by the situation model. The presence of overlapping situation models acts like a bridge between memories for surface features and the textbase to cross, and provides a mechanism by which context-independent representations can enhance repetition effects when a situation model is well developed. In essence, overlapping situation models support transfer at the level of the surface form and textbase, rather than suppressing them.

What experimental tasks meet the conditions defined by bars A and B? An example of task-relevant, overlapping situations (bar A) is reading normal texts for comprehension. All models of text repetition predict large repetition effects for rereading of an identical text. According to the context-dependentrepresentation model, repetition effects for identical passages are large because the surface form, textbase, and situation model are identically repeated and the texts are processed in the same manner. Repetition effects are also expected when the situation model is repeated in the second text, but elements of the surface form or textbase are changed. This occurs when the second reading is a paraphrase (Levy et al., 1993; Raney et al., 2000), a translation (Raney et al., 1996), or a semantically related text that activates the episode described by the original text (Levy et al., 1993; Levy et al., 1995). Repetition effects in these cases should be smaller than for identically repeated texts because there is only partial overlap of the surface form and textbase, and potentially the situation model.

When a situation model is task relevant but the texts do not refer to the same situations (bar B), the contextdependent representation model predicts little repetition benefit. An example of this condition is reading two normal texts about different topics. Reading one text does not facilitate processing of a second text when the topics are distinct, even if the words overlap (Levy et al., 1993; Levy et al., 1995; Raney et al., 1997). A second example is reading a scrambled version of a text followed by a normal version of the text with instructions to comprehend the passages (Carlson et al., 1991; Levy \& Burns, 1990). When comprehension of a scrambled text is required, readers will try to develop a situation model. Depending on the degree of scrambling, the situation model will be incomplete or incorrect and will not match the subsequently read normal text (Levy \& Burns, 1990; Levy et al., 1991; Masson, 1995). The greater the mismatch, the smaller the repetition effect. Thus, scrambling at the word level, which substantially limits the formation of a situation model, should produce less repetition benefit for a subsequent normal text than scrambling at the paragraph level, which allows more situation-level comprehension.

When repetition effects are based on complete, taskrelevant situation models (bars A and B), repetition effects should be larger for rereading a text than for reading a second, related text because rereading reinstates the same situation model whereas a related text can only reinstate the portions of the situation model that are repeated or referenced. Levy et al. (1993; Levy et al., 1995) found large transfer benefits for related passages that were continuous sections of a novel. Their related texts were highly similar and referred to common characters, 
events, and goals. Raney et al. (1997) defined related texts as thematic overlap between a few sentences in short texts. Their related texts discussed common issues (e.g., the creation of new species), but were about different topics (e.g., animals vs. plants) and were not continuations of a single story. Thus, smaller transfer effects should be found in Raney et al. (1997) than in Levy et al. (1993; Levy et al., 1995). Beyond differences in magnitude, the factors that influence rereading benefits and transfer benefits are expected to be the same. I know of no studies that directly compare rereading benefits to transfer benefits.

Bars C and D of Figure 1 represent conditions in which situation models are not task relevant or cannot be well formed. Here the prediction is that intermediatesized repetition effects will occur if there is substantial repetition of the surface form or textbase. The error bars indicate that repetition effects will vary in size on the basis of the amount of overlap. Repetition effects should increase as the degree of overlap between surface features and textbases increases. When attention is directly focused on these levels of representation, repetition effects could be large.

Even with appropriate task demands, repetition effects are expected to be smaller than when facilitated by a well-formed, task-relevant situation model (bars A and B). When developing a situation model is secondary to the task or a complete situation model cannot be formed, the potential for overlapping situation models should produce an automatic benefit because it is difficult (or impossible) to completely ignore the meaning of a text while reading (Levy et al., 1991; Masson, 1995). As stated earlier, facilitation based on overlapping surface forms, textbases, and situation models is expected to combine and therefore increase the magnitude of repetition effects. As a result, repetition effects are predicted to be larger when situation models overlap than when they do not overlap, even if the task does not emphasize development of a situation model or if abnormal texts are read (e.g., scrambled or incoherent texts).

What experimental tasks meet the conditions represented by bars $C$ and $D$ ? An example that could include situation model overlap (bar C) is proofreading texts without comprehension demands (Singer \& Halldorson, 1996). Singer and Halldorson found that inference-based elements of the situation model were markedly reduced when subjects were instructed to proofread texts without comprehension requirements. In this example the situation model is incomplete and it is not the focus of the task. A second example is rereading normal or scrambled texts with instructions to pronounce each word correctly and ignore overall meaning (e.g., Carlson et al., 1991; Carr et al., 1989). Attention was focused on wording in those studies, so repetition effects appeared to be context independent. A third example is provided by Levy's (2001) experiments using texts that were globally incoherent if the title was not presented. Omitting the title makes it difficult for readers to develop a coherent situation model. Levy (2001) found that if a text was read without a title and then reread with the title (no-title/title), smaller repetition effects were found than when the title was presented before each reading (title/title). The surface form and textbase matched in both conditions, but the situation model matched only in the title/title condition.

An example of no situation model overlap (bar D) is reading two different scrambled versions of a single text (Brown \& Carr, 1993; Masson, 1995; Whittlesea, 1990). Scrambling at the word level substantially reduces or eliminates the development of a coherent textbase and situation model, so the degree of overlap of surface form should largely control the magnitude of repetition effects. This leads to the expectation that reading two identical versions of a scrambled text will produce a larger repetition effect than reading two different scrambled versions. Although situation models cannot be developed properly for fully scrambled texts, it is certainly possible to develop a theme for a scrambled passage or to form linkages between successive words. Any theme or word linkages developed during the first reading of a scrambled text would not precisely match a new scrambled version. This mismatch would reduce repetition effects relative to an identically repeated scrambling. Levy's (2001) experiments using texts with or without titles provide a second example. Repetition effects were larger when the title was presented before each reading (title/title) than when the title was presented only before the first reading (title/no-title) or not presented before either reading (no-title/no-title). The situation models did not match in the title/no-title or no-title/no-title conditions; therefore, repetition effects were smaller than in the title/title condition.

The prior examples bring up an important pointtrying to construct a situation model or some type of theme is a normal, automatic component of reading (Gernsbacher, 1990; Kintsch, 1998), as is processing words in normal or scrambled texts (Levy et al., 1991; Masson, 1995). Comprehension processes involved in the development of situation models are not turned on in one task and shut off in another task. Properties of the task, including the stimuli, do, however, influence the degree to which situation models are formed and, hence, applied (Gernsbacher, 1990; Glenberg et al., 1987; Graesser et al., 1997; Kintsch, 1998; O'Brien \& Albrecht, 1992; Singer \& Halldorson, 1996; Zwaan, 1994; Zwaan et al., 1995; Zwaan \& Radvansky, 1998).

The context-dependent representation model can be extended to explain repetition effects when creation of a situation model would be beneficial, but is not completed. Examples of this circumstance include poor readers and children who are learning to read, especially if the text is difficult. For poor readers and children, processing the surface form and textbase is difficult; therefore, extra processing resources must be focused on these levels. This could lead to repetition effects that reflect development and overlap of the surface form and textbase more so than the situation model, which is likely to be incomplete (Faulkner \& Levy, 1994; Oakhill, 1994; Oakhill, Cain, \& Yuill, 1998; Rashotte \& Torgesen, 
1985; Samuels, 1979). This particular example is difficult to classify into one of the groups depicted in Figure 1 . Given that a situation model is task relevant, it could fit bars A and B. Given that a situation model is poorly formed, it could fit bars $\mathrm{C}$ and $\mathrm{D}$. The example could be categorized by evaluating memory for the surface form, textbase, and situation model. If the situation information is not accessible or is incomplete (as expected on the basis of past research), the example would be classified as fitting bars $C$ and $D$ because surface features and the textbase would be used to guide retrieval. The situation model actually becomes secondary. The point here is that not all examples can be easily classified without knowing how the task is performed and what information is included in memory. (See Bates, Masling, \& Kintsch, 1978; Fletcher, 1994; Fletcher \& Chrysler, 1990; Kintsch, 1998; Kintsch \& Bates, 1977; Kintsch et al., 1990; Sachs, 1967; and van Dijk \& Kintsch, 1983, for research demonstrating differential memory strengths for the three levels of text representation.)

The model also supports predictions of repetition effects when information is not in text format. One instance of this would be presenting information pictorially during the first encounter and in text format during the second encounter. If the situation depicted in the picture matches the situation described in the text, then seeing the picture should facilitate processing the text. As with text, the degree to which the picture is understood should influence the degree of transfer. If the picture depicts an event or information that can be clearly understood, then the picture's situation model should be used to guide transfer to the text. If the picture cannot be clearly understood, then the picture's situation model would not be expected to guide transfer. Instead, individual elements of the picture (i.e., its surface features) should be used to guide transfer. Thus, transfer based on nontext information is expected to follow the same rules as transfer between texts. Conducting such an experiment would provide a useful test of the model.

\section{EXPLAINING CONTRADICTIONS IN PAST RESEARCH}

Past text repetition studies demonstrate many seemingly inconsistent results. The context-dependent representation model explains these inconsistencies on the basis of differences in text representations as a function of task requirements. Tasks that emphasize wording (e.g., reading aloud) will enhance memory for the surface form and textbase, whereas tasks that emphasize general meaning (e.g., comprehension and pleasantness judgments) will enhance memory for the situation (Carlson et al., 1991; Jacoby et al., 1992; Levy et al., 1995; Tardif \& Craik, 1989). A good example of this is provided by comparing Carr et al. (1989) with Levy and Burns (1990).

Recall that Carr et al. (1989) and Levy and Burns (1990, Experiment 3) had subjects read a text in normal form or with word order scrambled, then reread the same text or the opposite version. Carr et al. (1989) found repetition effects in each condition, whereas Levy and Burns found little or no repetition effects when a scrambled text was read after a normal text or vice versa. Carr et al. (1989) instructed readers to read aloud (emphasizing pronunciation of individual words) and ignore meaning. Consequently, the surface form and textbase were emphasized and the situation model was minimized (corresponding to bars $\mathrm{C}$ and $\mathrm{D}$ of Figure 1). This produced context-independent repetition effects. Levy and Burns instructed readers to read silently and to try and construct meaning even for the scrambled texts. Here the situation model was emphasized. This produced context-dependent repetition effects. Carlson et al. (1991) confirmed that changing the task demands changes the reader's focus of attention and alters the pattern of repetition effects.

The context-dependent representation model can also be used to explain the trend reported by Levy and Burns (1990), but not by Carr et al. (1989), for normal-scrambled pairs to produce slightly more repetition benefit than scrambled-normal pairs. Reading a normal text first would produce a situation model that could be used to help make sense of the scrambled text (also see Levy, 2001). This would enhance the repetition effect. In contrast, a coherent situation model cannot be fully developed during reading of a completely scrambled text, so transfer based on situation overlap would be limited. Because Carr et al.'s (1989) experiments emphasized wording, not situation model development, this same trend would not be expected.

\section{FUTURE RESEARCH}

In its present form, the proposed model is able to account for the majority of text repetition effects found in past research. Even so, the model only represents a starting point for understanding how context dependence influences text memory and repetition benefits. Aspects of the model that need refinement are pointed out here with the intention of stimulating future research that will help clarify the model.

One area needing refinement is the difference in context dependence between the surface form and the textbase. The textbase is generally described as a set of independent propositions that are subsequently linked together to form a macrostructure (Kintsch, 1974; Kintsch \& van Dijk, 1978). Because propositions can be processed independently, they can be viewed as context independent. On the other hand, the textbase represents the semantic content of the text, and the situation model expands upon the textbase. Given that the textbase and situation model overlap, the textbase might be viewed as context dependent. The bulk of the research performed so far indicates that the textbase can be modified without eliminating repetition effects (Levy et al., 1993; Levy et al., 1995; Raney et al., 1996; Raney et al., 2000), which is consistent with context-independent represen- 
tation. If context dependence is a continuum, the textbase might lie in the middle range of this continuum. Additional research is needed to resolve this issue.

Another topic that needs further exploration is negative transfer between texts (i.e., reading one text makes a subsequent text more difficult to process). The model was designed to explain positive transfer (i.e., facilitation), but evidence also exists that reading one text can have a negative influence on a second text (Levy et al., 1997; Levy et al., 1995). In Levy's studies, readers read two passages that shared vocabulary (surface features) but not content. In this case, reading the first passage slowed reading of the second passage. These effects were small and inconsistent, but they are still important to explain. Levy and colleagues referred to these negative transfer effects as inhibitory effects, but this outcome also could reflect passive interference between the first text and the second text.

One way to explain negative transfer is to think of passages that share words but not content as similar to homographs (words with multiple meanings, such as bank). Rayner, Pacht, and Duffy (1994) showed that when a homograph is presented twice in a text and the meaning is changed from the first to the second encounter, reading time slows during the second encounter. Something similar could happen at the text level. If many words from the first and second texts match, then words from the second text might lead to reinstatement of meaning elements from the first text (see Levy et al., 1995, for a similar explanation). Retrieving mismatched meanings would slow processing of the second text. This reinstatement process might function much like a resonance process in anaphor resolution (O'Brien et al., 1997). Although speculative, this explanation leads to testable predictions. For example, the more the texts match at the word level, the greater the negative transfer should be. In addition, substantial negative transfer should occur only if the words are contextually bound to the texts-that is, when situation models are well developed and task relevant. Negative transfer would not be expected when attention is focused on wording (e.g., reading out loud two scrambled passages that share wording). Even though this explanation is speculative, it illustrates that the model can be used to generate unique predictions for future research.

Another important area for future research is individual differences. We know that skilled readers develop more complete situation models than do poor readers and children (Faulkner \& Levy, 1994; Oakhill, 1994; Rashotte \& Torgesen, 1985; Samuels, 1979). We also know that the link between the surface form and the overall meaning of a text is not as strong for poor readers as for skilled readers (Bourassa et al., 1998). These findings lead to the prediction that the degree of context dependence will be less in poor readers and children than in skilled readers. A similar situation might exist for skilled readers as a function of domain-specific knowledge. For example, Tardieu, Ehrlich, and Gyselinck (1992) showed that do- main experts form better situation models than do domain novices (also see Kintsch \& Franzke, 1995). In terms of repetition effects, poor readers, children, and domain novices might produce more context-independent repetition effects than skilled readers, adults, and domain experts. Given that situation models play a key role in the context-dependent representation model, enhancing our understanding of situation model development will support further refinement of the proposed model (see van Oostendorp \& Goldman, 1999, for current reviews of text representation and situation models).

\section{CONCLUSIONS}

In this article, I (1) reviewed abstract and episodic models of text repetition effects, (2) described the research base supporting these two models, and (3) proposed a new model that integrates context-independent representations and context-dependent representations into a single text memory. To summarize, according to the contextdependent representation model, a text's surface form and textbase are represented in a context-independent manner. Context-independent representations support repetition effects that transfer across linguistic contexts. A coherent task-relevant situation model binds the surface features and the textbase to the text representation, which leads to context-dependent representations. Contextdependent representations limit repetition effects to conceptually similar linguistic contexts.

As knowledge about text comprehension and memory is enhanced, the assumptions and predictions of the model can be refined. As is, the context-dependent representation model provides a theoretically meaningful organization for explaining many seemingly inconsistent past results. The model can also serve as a useful guide for future research aimed at understanding text repetition effects as well as text comprehension and memory in general.

\section{REFERENCES}

AltarribA, J. (1992). The representation of translation equivalents in bilingual memory. In R. J. Harris (Ed.), Cognitive processing in bilinguals (pp. 157-174). Amsterdam: Elsevier.

Altarriba, J., Kroll, J. F., Sholl, A., \& Rayner, K. (1996). The influence of lexical and conceptual constraints on reading mixed-language sentences: Evidence from eye fixations and naming times. Memory \& Cognition, 24, 477-492.

Bates, E., Masling, M., \& Kintsch, W. (1978). Recognition memory for aspects of dialogue. Journal of Experimental Psychology: Human Learning \& Memory, 3, 187-197.

Bourassa, D. C., Levy, B. A., Dowin, S., \& CASey, A. (1998). Transfer effects across contextual and linguistic boundaries: Evidence from poor readers. Journal of Experimental Child Psychology, 71, 45-61.

Bowers, J. S. (2000). In defense of abstractionist theories of repetition priming and word identification. Psychonomic Bulletin \& Review, 7, 83-99.

BRANSFORD, J. D. (1979). The role of prior knowledge. In J. D. Bransford (Ed.), Human cognition: Learning, understanding, and remembering (pp. 129-165). Belmont, CA: Wadsworth.

Brown, J. S., \& CARR, T. H. (1993). Limits on perceptual abstraction 
in reading: Asymmetric transfer between surface forms differing in typicality. Journal of Experimental Psychology: Learning, Memory, \& Cognition, 19, 1277-1296.

Carlson, L., Alejano, A., \& Carr, T. H. (1991). The level of focal attention hypothesis in oral reading: Influences of strategies on the context specificity of lexical repetition effects. Journal of Experimental Psychology: Learning, Memory, \& Cognition, 17, 924-931.

Carr, T. H., \& Brown, J. S. (1990). Perceptual abstraction and interactivity in repeated oral reading: Where do things stand? Journal of Experimental Psychology: Learning, Memory, \& Cognition, 16, 731-738.

Carr, T. H., Brown, J. S., \& Charalambous, A. (1989). Repetition and reading: Perceptual encoding mechanisms are very abstract but not very interactive. Journal of Experimental Psychology: Learning, Memory, \& Cognition, 15, 763-778.

Carr, T. H., Dagenbach, D., VAn Wieren, D., Carlson-Radvansky, L., Alejano, A., \& Brown, J. (1994). Acquiring general knowledge from specific episodes of experience. In C. Umiltà \& M. Moscovitch (Eds.), Attention and performance XVI: Conscious and nonconscious information processing (pp. 697-724). London: Erlbaum.

DE Groot, A. M. B., \& NAS, G. (1991). Lexical representation of cognates and noncognates in compound bilinguals. Journal of Memory \& Language, 30, 90-123.

FAulkner, H. J., \& Levy, B. A. (1994). How text difficulty and reader skill interact to produce differential reliance. Journal of Experimental Child Psychology, 58, 1-24.

FAulKner, H. J., \& LEVy, B. A. (1999). Fluent and nonfluent forms of transfer in reading: Words and their message. Psychonomic Bulletin \& Review, 6, 111-116.

Fletcher, C. R. (1994). Levels of representation in memory for discourse. In M. A. Gernsbacher (Ed.), Handbook of psycholinguistics (pp. 589-607). San Diego: Academic Press.

Fletcher, C. R., \& Chrysler, S. T. (1990). Surface forms, textbases, and situation models: Recognition memory for three types of textual information. Discourse Processes, 13, 175-190.

Gernsbacher, M. A. (1990). Language comprehension as structure building. Hillsdale, NJ: Erlbaum.

Glanzer, M., \& Nolan, S. D. (1986). Memory mechanisms in text comprehension. In G. Bower (Ed.), The psychology of learning and motivation: Advances in research and theory (pp. 275-317). San Diego: Academic Press.

Glenberg, A. M., Meyer, M., \& Lindem, K. (1987). Mental models contribute to foregrounding during text comprehension. Journal of Memory \& Language, 26, 69-83.

Goldman, S. R., \& SaUl, E. U. (1990). Flexibility in text processing: A strategy competition model. Learning \& Individual Differences, $\mathbf{2}$, 181-219.

Graesser, A. C., Millis, K. K., \& ZwaAn, R. A. (1997). Discourse comprehension. Annual Review of Psychology, 48, 163-189.

Horton, K. D. (1985). The role of semantic information in reading spatially-transformed text. Cognitive Psychology, 17, 66-88.

Humphreys, G. W., Besner, D., \& Quinlan, P. T. (1988). Event perception and the word repetition effect. Journal of Experimental Psychology: General, 117, 51-67.

JACOBY, L. L. (1983). Perceptual enhancement: Persistent effects of an experience. Journal of Experimental Psychology: Learning, Memory, \& Cognition, 9, 21-38.

JACOBY, L. L., Levy, B. A., \& Steinbach, K. (1992). Episodic transfer and automaticity: Integration of data-driven and conceptually-driven processing in reading. Journal of Experimental Psychology: Learning, Memory, \& Cognition, 18, 15-24.

Johnson-LaIrd, P. N. (1983). Mental models: Towards a cognitive science of language, inference, and consciousness. Cambridge, MA: Harvard University Press.

KINTSCH, W. (1974). The representation of meaning in memory. Hillsdale, NJ: Erlbaum.

KINTSCH, W. (1988). The role of knowledge in discourse comprehension: A construction-integration model. Psychological Review, 95, 163-182.

Kintsch, W. (1998). Comprehension: A paradigmfor cognition. Cambridge: Cambridge University Press.

Kintsch, W., \& BATES, E. (1977). Recognition memory for statements in a classroom lecture. Journal of Experimental Psychology: Human Learning \& Memory, 3, 150-159.
Kintsch, W., \& Franzke, M. (1995). The role of background knowledge in the recall of news story. In R. Lorch \& E. O'Brien (Eds.), Sources of coherence in reading (pp. 321-333). Hillsdale, NJ: Erlbaum.

Kintsch, W., \& VAn DiJK, T. A. (1978). Toward a model of text comprehension and production. Psychological Review, 85, 363-394.

Kintsch, W., Welsch, D., Schmalhofer, F., \& Zimny, S. (1990). Sentence memory: A theoretical analysis. Journal of Memory \& Language, 29, 133-159.

Kolers, P. A. (1975). Specificity of operations in sentence recognition. Cognitive Psychology, 1, 289-306.

Kolers, P. A. (1976). Reading a year later. Journal of Experimental Psychology: Human Learning \& Memory, 2, 554-565.

Kolers, P. A., \& Ostry, D. J. (1974). Time course of loss of information regarding pattern analyzing operations. Journal of Verbal Learning \& Verbal Behavior, 13, 599-612.

Kroll, J. F., \& DE Groot, A. M. B. (1997). Lexical and conceptual memory in the bilingual: Mapping form to meaning in two languages. In A. M. B. de Groot \& J. F. Kroll (Eds.), Tutorials in bilingualism: Psycholinguistic perspectives (pp. 169-200). Mahwah, NJ: Erlbaum.

Levy, B. A. (1983). Proofreading familiar text: Constraints on visual processing. Memory \& Cognition, 11, 1-12.

LEVY, B. A. (1993). Fluent rereading: An indirect indicator of reading skill development. In P. Graf \& M. E. J. Masson (Eds.), Indirect memory: New directions in cognition, development, and neuropsychology (pp. 49-73). Hillsdale, NJ: Erlbaum.

LeVy, B. A. (2001). Text processing: Memory representations mediate fluent reading. In M. Naveh-Benjamin, M. Moscovitch, \& H. L. Roediger III (Eds.), Perspectives on human memory and cognitive aging: Essays in honourof Fergus Craik (pp. 83-98). New York: Psychology Press.

Levy, B. A., Abello, B., \& Lysynchuk, L. (1997). Transfer from word training to reading in context: Gains in reading fluency and comprehension. Learning Disability Quarterly, 20, 173-188.

Levy, B. A., Barnes, L., \& Martin, L. (1993). Transfer of fluency across repetitions and across texts. Canadian Journal of Experimental Psychology, 47, 401-427.

Levy, B. A., \& Begin, J. (1984). Proofreading familiar text: Allocating resources to perceptual and conceptual processes. Memory \& Cognition, 12, 621-632.

Levy, B. A., \& Burns, K. I. (1990). Reprocessing text: Contributions from conceptually driven processes. Canadian Journal of Psychology, 44, 465-482.

Levy, B. A., Campsall, J., Browne, J., Cooper, D., Waterhouse, C., \& WiLson, C. (1995). Reading fluency: Episodic integration across texts. Journal of Experimental Psychology: Learning, Memory, \& Cognition, 21, 1169-1185.

Levy, B. A. Di Persio, R., \& Hollingshead, A. (1992). Fluent rereading: Repetition, automaticity, and discrepancy. Journal of Experimental Psychology: Learning, Memory, \& Cognition, 18, 957-971.

LeVy, B. A., \& KIRSNER, K. (1989). Reprocessing text: Indirect measures of word and message level processes. Journal of Experimental Psychology: Learning, Memory, \& Cognition, 15, 407-417.

Levy, B. A., Masson, M. E. J., \& ZoubeK, M. A. (1991). Rereading text: Words and their context. Canadian Journal of Psychology, 45, 492-506.

Long, D. L., \& Chong, J. L. (2001). Comprehension skill and global coherences: A paradoxical picture of poor comprehenders' abilities. Journal of Experimental Psychology: Learning, Memory, \& Cognition, 27, 1424-1429.

Long, D. L., Oppy, B. J., \& SeEly, M. R. (1997). Individual differences in readers' sentence and text-level representation. Journal of Memory \& Language, 36, 129-145.

Masson, M. E. J. (1995). Episodically enhanced comprehension fluency. In J. Henderson, M. Singer, \& F. Ferreira (Eds.), Reading and language processing (pp. 300-337). Hillsdale, NJ: Erlbaum.

Masson, M. E. [J.], \& Freedman, L. (1990). Fluent identification of repeated words. Journal of Experimental Psychology: Learning, Memory, \& Cognition, 16, 355-373.

Millis, K., \& SimON, S. (1994). Rereading scientific texts: Changes in resource allocation. In H. van Oostendorp \& R. Zwaan (Eds.), Naturalistic text comprehension (pp. 115-133). Norwood, NJ: Ablex.

Monsell, S. (1985). Repetition and the lexicon. In A. W. Ellis (Ed.), 
Progress in the psychology of language (Vol. 2, pp. 147-196). London: Erlbaum.

Monsell, S. (1987). Non-visual orthographic processing and the orthographic lexicon. In M. Coltheart (Ed.), Attention and performance XII (pp. 299-326). London: Erlbaum.

Morton, J. (1969). Interaction of information in word recognition. Psychological Review, 76, 165-178.

Morton, J. (1979). Facilitation in word recognition: Experiments causing change in the logogen model. In P. A. Kolers, M. E. Wrolstad, \& H. Bouma (Eds.), Processing models of visible language (pp. 259-268). New York: Plenum.

OAKHILL, J. (1994). Individual differences in children's text comprehension. In M. A. Gernsbacher (Ed.), Handbook of psycholinguistics (pp. 821-848). San Diego: Academic Press.

OAKHill, J., CAIN, K., \& Yuill, N. (1998). Individual differences in children's comprehension skill: Toward an integrated model. In C. Hulme \& R. M. Joshi (Eds.), Reading and spelling (pp. 343-367). Mahwah, NJ: Erlbaum.

O'Brien, E., \& Albrecht, J. (1992). Comprehension strategies in the development of a mental model. Journal of Experimental Psychology: Learning, Memory, \& Cognition, 18, 777-784.

O'Brien, E., Raney, G. E., Albrecht, J., \& Rayner, K. (1997). Processes involved in the resolution of explicit anaphors. Discourse Processes, 23, 1-24.

Pollatsek, A., Raney, G. E., LaGasse, L., \& Rayner, K. (1995). The use of information below fixation in reading and in visual search. In J. Henderson, M. Singer, \& F. Ferreira (Eds.), Reading and language processing (pp. 51-72). Hillsdale, NJ: Erlbaum.

RANEY, G. E. (1993). Monitoring changes in cognitive load during reading: An event-related brain potential and reaction time analysis. Journal of Experimental Psychology: Learning, Memory, \& Cognition, 19, 51-69.

Raney, G. E. Atilano, R, \& Gomez, L. (1996, November). Language representation and bilingual reading. Paper presented at the meeting of the Psychonomic Society, Chicago.

Raney, G. E., Minkoff, S., \& Therriault, D. (1997, November). Using interleaved reading to study text processing. Paper presented at the meeting of the Psychonomic Society, Philadelphia.

Raney, G. E., Obeidallah, S., \& Miura, T. (2002). Text comprehension in bilinguals: Integrating perspectives on language representation and text processing. In R. Heredia \& J. Altarriba (Eds.), Bilingual sentence processing (pp. 165-186). Amsterdam: Elsevier.

RANEY, G. E., \& RAYNER, K. (1993). Event-related brain potentials, eye movements, and reading. Psychological Science, 4, 283-286.

RANEY, G. E., \& RAYNER, K. (1995). Word frequency effects and eye movements during two readings of a text. Canadian Journal of Experimental Psychology, 49, 151-172.

Raney, G. E., Therriault, D., \& Minkoff, S. (2000). Repetition effects from paraphrased text: Evidence for an integrated representation model of text representation. Discourse Processes, 29, 61-81.

Rashotte, C. A., \& Torgesen, J. K. (1985). Repeated reading and reading fluency in learning disabled children. Reading Research Quarterly, 20, 180-202.

RAYNer, K., PACht, J. M., \& Duffy, S. A. (1994). Effects of prior encounter and discourse bias on the processing of lexically ambiguous words: Evidence from eye fixations. Journal of Memory \& Language, 33, 527-544.

RAYNer, K., \& Pollatsek, A. (1989). The psychology of reading. Englewood Cliffs, NJ: Prentice-Hall.

Rayner, K., Raney, G. E., \& Pollatsek, A. (1995). Eye movements and discourse processing. In R. Lorch \& E. O'Brien (Eds.), Sources of coherence in reading (pp. 9-36). Hillsdale, NJ: Erlbaum.

Roediger, H. L., III (1990). Implicit memory: Retention without remembering. American Psychologist, 45, 1043-1056.

RUECKL, J. G. (1990). Similarity effects in word and pseudoword repetition priming. Journal of Experimental Psychology: Learning, Memory, \& Cognition, 16, 374-391.

RugG, M. D. (1990). Event-related brain potentials dissociate repetition effects of high- and low-frequency words. Memory \& Cognition, 18, 367-379.

SACHS, J. S. (1967). Recognition memory for syntactic and semantic aspects of connected discourse. Perception \& Psychophysics, 2, 437-442.
Salasoo, A. (1986). Cognitive processes in oral and silent reading comprehension. Reading Research Quarterly, 21, 59-69.

SAmuels, S. J. (1979). The method of repeated readings. The Reading Teacher, 32, 403-408.

Scarborough, D. L., Cortese, C., \& Scarborough, H. S. (1977). Frequency and repetition effects in lexical memory. Journal of Experimental Psychology: Human Perception \& Performance, 3, 1-17.

Singer, M., \& Halldorson, M. (1996). Constructing and validating motive bridging inferences. Cognitive Psychology, 30, 1-38.

Tardieu, H., Ehrlich, M. F., \& Gy SelincK, V. (1992). Levels of representation and domain-specific knowledge in comprehension of scientific texts. Language \& Cognitive Processes, 7, 335-351.

TARdif, T., \& CraiK, F. I. M. (1989). Reading a week later: Perceptual and conceptual factors. Journal of Memory \& Language, 28, 107-125.

Tenpenny, P. L. (1995). Abstractionist versus episodic theories of repetition priming and word identification. Psychonomic Bulletin \& Review, 2, 339-363.

van DiJK, T. A., \& KInTsCh, W. (1983). Strategies of discourse comprehension. New York: Academic Press.

van Oostendorp, H., \& Goldman, S. R. (1999). The construction of mental representations during reading. Hillsdale, NJ: Erlbaum.

Whittlesea, B. W. A. (1990). Perceptual encoding mechanisms are tricky but may be very interactive: Comment on Carr, Brown, and Charalambous (1989). Journal of Experimental Psychology: Learning, Memory, \& Cognition, 16, 727-730.

Woltz, D. J. (1990). Repetition of semantic comparisons: Temporary and persistent priming effects. Journal of Experimental Psychology: Learning, Memory, \& Cognition, 16, 392-403.

ZWAAN, R. A. (1994). Effect of genre expectations on text comprehension. Journal of Experimental Psychology: Learning, Memory, \& Cognition, 20, 1196-1207.

Zwaan, R. A., Langston, M. C., \& Graesser, A. C. (1995). The construction of situation models in narrative comprehension: An eventindexing model. Psychological Science, 6, 282-297.

ZWAAN, R A., \& RAdVANSKy,G. A (1998). Situation models in language comprehension and memory. Psychological Bulletin, 123, 162-185.

\section{NOTES}

1. Levy et al. (1995) defined related texts (their message overlap condition) as "a pair of sequential sections from a novel" (p. 1171). It is debatable whether these texts should be defined as separate texts or as continuations of a single story. By their definition, any text could be divided in half and described as two texts. Research by Pollatsek, Raney, LaGasse, and Rayner (1995) and Rayner et al. (1995) demonstrates that reading speed increases as a reader progresses through a text. This could lead to faster overall reading times for the "second text" in the related condition of Levy et al.'s (1995) study.

2. It is interesting to note that current models of monolingual and bilingual lexical representation postulate separate levels of representation for word forms and their meanings (see Kroll \& de Groot, 1997, for a review). This represents a hierarchical form of representation, which is inherently consistent with the finding of separate facilitation of word forms and word meanings (see Raney, Obeidallah, \& Miura, 2002, for further discussion of this point).

3. Earlier versions of the context-dependent representation model were referred to as the integrated-representation model.

4. The interleaved reading procedure was used to control the distance (number of sentences) between the first and second occurrences of the target words. Under normal reading circumstances, repetitions of words across two passages are typically farther apart (in time and number of sentences) than repetitions within a single passage. This could lead to small or nonexistent repetition effects for across-passage repetitions for reasons unrelated to the study. For example, as the distance between repetitions increases, more time is needed to link the first and second occurrence of the target words and repetition benefits decrease in size (O’Brien et al., 1997; Rayner et al., 1995).

(Manuscript received July 1, 1998; revision accepted for publication March 1, 2002.) 